\title{
SIMULATIONS OF CURRENTS IN X-BAND ACCELERATOR STRUCTURES USING 2D AND 3D PARTICLE-IN-CELL CODE*
}

\author{
Valery A. Dolgashev, Sami G. Tantawi ${ }^{\dagger}$, SLAC, Stanford, CA 94309, USA
}

\section{INTRODUCTION}

Accelerating gradient is one of the crucial parameters affecting design, construction and cost of next-generation linear accelerators. For a specified final energy, the gradient sets the accelerator length, and for a given accelerating structure and pulse repetition rate it determines power consumption. Accelerating gradients on the order of 100 $\mathrm{MV} / \mathrm{m}$ have been reached in short $(\sim 20 \mathrm{~cm})$ standing wave and traveling wave X-band accelerating structures $[1,2,3]$. But recent experiments have shown damage to traveling wave accelerating structures at gradients as low as $50 \mathrm{MV} / \mathrm{m}$ after 1000 hours of operation [4]. RF breakdown is a probable cause of this damage. An extensive experimental and theoretical program to determine a safe operating gradient for the Next Linear Collider (NLC) is under way in SLAC. The present work is a part of that program.

\subsection{RF breakdown}

We define rf breakdown as a phenomenon that abruptly and significantly changes transmission and reflection of the rf power directed to the structure under test. We distinguish breakdown from field emission and dark current. Dark currents have reproducible and monotonic (with respect to input power) behavior in spite their random space-time origin. There is evidence [4] that rf breakdown can damage the structure. RF breakdown is a complex phenomenon and its physics is yet to be understood. It includes the rf driven interaction of electrons, ions and neutral atoms, heating and melting of the metal surface etc. The short time scale of the breakdown $(\sim 5 \mathrm{~ns}-10 \mu \mathrm{s})$, its unpredictable starting time, and the random location of the breakdown site make it very difficult to observe its microscopic behavior. In contrast, we routinely detect and record external macroscopic parameters such as incident rf power, power reflected from the breakdown site, and power transmitted through it. We use other parameters, such as emitted light, $\mathrm{X}$-rays and harmonics of the working frequency to obtain more information about the physics of the breakdown phenomenon. Other parameters are the electron currents that exit from the beam pipe of the accelerating structure.

\subsection{Simulations}

We use a simplified physical model to simulate the breakdown. We assume that part of the structure surface starts emitting electron and ion currents at a predetermined time (the physics of this emission are not considered here).

\footnotetext{
* This work was supported by the U.S. Department of Energy contract DE-AC03-76SF00515.

$\dagger$ Also with the Communications and Electronics Department, Cairo University, Giza, Egypt.
}

We adjust the emission models to resemble observed parameters (transmitted and reflected $\mathrm{rf}$ ) of the experimental data. We have chosen a commercial Particle-In-Cell (PIC) code MAGIC [6] for these simulations. This code is used at SLAC for 2D and 3D simulation of klystrons. Such features of the code as the capability for input of realistic geometries and developed diagnostics (of particle and field parameters) make it very useful for the simulations. Unfortunately, the chosen code does not let us use small emission areas. Traces left by breakdowns on metal surfaces have a size $\sim 10-100 \mu \mathrm{m}$ and the smallest mesh size in the PIC code is $\sim 1 \mathrm{~mm}$. Another limitation is the maximum size of the simulated structure. We have simulated up to 8 cells $(\sim 10 \mathrm{~cm})$ of $11.4 \mathrm{GHz}$ standing wave accelerating structure in 2D and 5 cells $(\sim 5 \mathrm{~cm})$ of traveling wave structure in $3 \mathrm{D}$, while real structures have lengths up to $1.8 \mathrm{~m}$. The simulations were guided by comparison with measurements of breakdown in a simple rectangular waveguide. The breakdown process in a waveguide is both easier to measure and to simulate than breakdown in the complex geometry of an accelerating structure.

\section{BREAKDOWN IN A WAVEGUIDE}

\subsection{Experiment}

As part of the breakdown research program we studied rf breakdown in a rectangular waveguide.

Its width was reduced to $1.33 \mathrm{~cm}$ (in comparison with a width of $2.29 \mathrm{~cm}$ for WR90) over a length of $6 \mathrm{~cm}$ in order to enhance the electric field by lowering the group velocity to $0.18 \mathrm{c}$, and force the breakdown to occur in a this area. The height of the waveguide is $1.02 \mathrm{~cm}$. We subjected the waveguide to rf power up to $120 \mathrm{MW}$ with pulse widths up to $1.2 \mu \mathrm{s}$. We recorded incident, transmitted and reflected rf power; intensity of light emission and its spectrum; intensity of X-rays; and harmonics of the working frequency of $11.424 \mathrm{GHz}$. Harmonics were present in the klystron output as well as being generated by rf breakdown. We list some general conclusions about breakdown behavior deduced from the results of this experiment:

1. Transmitted power has a repeatable shape: it drops off to zero with an amplitude proportional to $e^{\frac{-\left(t-t_{s}\right)^{2}}{2 \tau^{2}}}$ (Gaussian-like). Here $t_{s}$ is breakdown start time. The rf pulse starts at $t=0$. The range of the drop off time constant $\tau$ is between 10 and 200 ns. For a preprocessed waveguide $\tau$ is $\sim 30 \mathrm{~ns}$. Here preprocessing means steady running at $\sim 100 \mathrm{MW}$ of $\mathrm{rf}$ power through the waveguide with a short pulse length $\sim 300 \mathrm{~ns}$.

2. Transmission does not recover for several microseconds after the breakdown. 
3. During the Gaussian drop off the transmitted and often the reflected signals have a few oscillations.

4. Up to $90 \%$ of the incident rf energy is absorbed after $t_{s}+2 \tau$. Breakdowns in a preprocessed waveguide absorb (on average) less energy than otherwise.

5. RF transmission fully recovers after the main rf pulse has been off for several milliseconds.

6. Light (emitted from the breakdown site) lasts for several microseconds after the rf pulse.

7. Spectral lines of the light are mostly from neutral copper atoms ( $\mathrm{Cu} \mathrm{I})$ with traces of $\mathrm{Cu}$ II ions and hydrogen.

8. Breakdowns tend to occur on sequences of $\mathrm{rf}$ pulses. Subsequent breakdowns most probably have a shorter starting time $t_{s}$ than the first breakdown of a sequence.

9. Breakdowns at $\mathrm{rf}$ high power $(\sim 100 \mathrm{MW})$ and short pulse length $(\sim 300 \mathrm{~ns})$ decrease dark currents, and lower power and a longer pulse (more than 400 ns) increases dark currents. We think that the level of dark currents indicates the degree of metal surface damage.

10. Breakdown has no detectable effect on laser light $(632.8 \mathrm{~nm})$ passing through the waveguide .

11. In most events, the 3rd harmonic $(34.272 \mathrm{GHz})$ signal from the klystron transmitted through the breakdown site is shut off by the breakdown.

12. Breakdown produces a 3rd harmonic of the klystron signal, and, probably, higher harmonics.

13. Characteristic size of the damaged spots on the surface of the waveguide is $\sim 10-100 \mu \mathrm{m}$.

\subsection{Simulations}

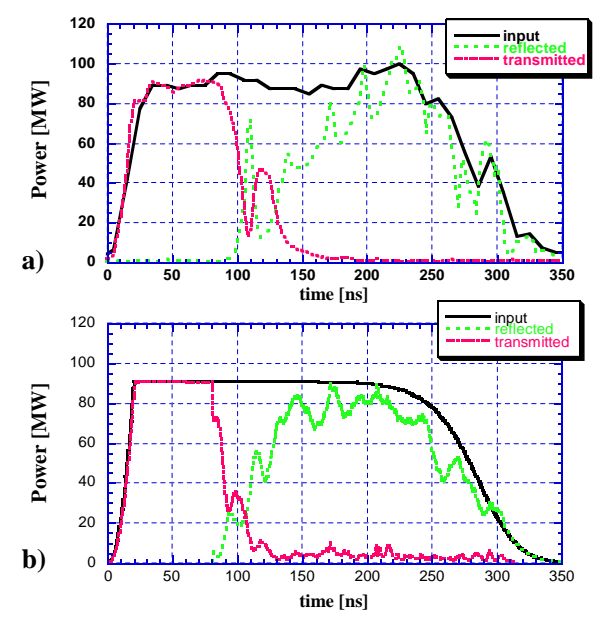

Figure 1: Incident, reflected and transmitted rf power for a breakdown in the waveguide. a) a measurement of a typical breakdown in the preprocessed waveguide. b) 3D PIC simulations, with an emitting spot size $4 \mathrm{~mm} \times 4 \mathrm{~mm}$, an electron current of $7 \mathrm{kA}$, and a copper ion current of $30 \mathrm{~A}$.

We performed 3D PIC simulations of the breakdown in the waveguide. The waveguide model has the same cross section as the experiment and a length of $6 \mathrm{~cm}$. There is no reflection from the waveguide's ends for the TE01 mode.
We simulated breakdown by creating emission spots on the broad walls of the waveguide. We applied space-charge limited emission of electrons. For that we used the builtin code feature EMISSION EXPLOSIVE. For copper ions we used a beam generated at the same area with a predetermined current density and initial velocity distribution (BEAM model in MAGIC). We changed such parameters as size and position of the emitting spots, input power, initial characteristics of the ion beam, and density of a neutral gas. We varied the size of the emitting spots from $1.6 \mathrm{~mm} \times 1.6 \mathrm{~mm}$ up to $1 \mathrm{~cm} \times 4 \mathrm{~cm}$. From this numerical experiment we came to the conclusions listed below.

1. The major energy exchange between incident rf fields and particles comes from the interaction of the rf electric fields with electrons (not with ions). Electrons cross the waveguide in a short time ( $\sim$ rf period).

2. The electron current must be several kA to significantly effect the rf power transmission. If we take into account the size of the damaged area, the current density must be in the order of $10^{7} \mathrm{~A} / \mathrm{cm}^{2}$. Space chargelimited emission of electrons without ions cannot produce and sustain such current densities.

3. Ion currents must be 10 to $100 \mathrm{~A}$ to disrupt transmitted power. An initial energy up to $50 \mathrm{eV}$ does not change the ion dynamics. The space charge fields of the ions compensates the electron space charge fields. This compensation allows the generation of $\mathrm{kA}$ of electron current. The time constant of the drop off of the transmitted power $\tau$ is 10 to $20 \mathrm{~ns}$ and is related mostly to the process of filling the waveguide gap with copper ions.

4. Without electrons the ions do not move significantly during the $\mathrm{rf}$ pulse. In the presence of space-charge limited electron flow, the ion beam crosses the waveguide in about $30 \mathrm{~ns}$ at $80 \mathrm{MW}$ of input power. The oscillating space charge field of the electrons adds a dc component to the rf electric field that accelerates the ions.

5. A significant portion (50-80\%) of the emitted electrons and ions returns to the emitting spot and the surrounding area.

6. During the rf pulse, most electrons and ions are confined to a beam with a cross-section area of about $1 \mathrm{~cm}^{2}$.

7. The transmitted and reflected power oscillates with a period 10-40 ns, determined by the ion-electron density.

8. The ion-electron current generates harmonics of the working frequency. The perturbation of the incident electric field due to these harmonics is on the order of $10 \%$.

9. Up to $50 \%$ of the input power can be absorbed by the ion-electron beam.

10. Up to $75 \%$ of the input power was absorbed by the ion-electron beam after we added some effects associated with the interaction of electrons with neutral copper atoms. 
A comparison between a signal from an typical actual breakdown and simulation with similar parameters is shown on Fig.1. The experience that we gained using the PIC code to understand waveguide breakdown gives us confidence in applying the same method to study breakdown in accelerating structures.

\section{ACCELERATING STRUCTURES}

\subsection{Traveling wave}

The characteristics of rf breakdown in traveling wave (TW) accelerating structures [5] are similar to those of waveguide breakdowns. The main similarities are: a drop off to zero of the transmitted rf power in tens of nanoseconds, with up to $80 \%$ of the incident rf energy absorbed after breakdown starts. We think that this analogy comes from some common characteristic of the waveguide and TW structures. Both have a broadband frequency response and are designed to transmit rf power. A breakdown that is localized in a limited volume or single cell does not change the ability of the structure to channel power to the breakdown site. We simulated 2D and 3D models of a TW accelerating structure. We used dimensions of a structure with an initial group velocity $0.05 \mathrm{c}$ that is currently under high power test at SLAC. We placed the emission spots on the iris of a structure cell. Results of PIC simulations of the accelerating structure lead to conclusions that are very similar to those for waveguides. We add some conclusions related to specifics of the accelerating structure below:

1. There is an asymmetry in the portion of electron current that exits through the beam apertures. Current is more likely to go toward the input coupler from the cell with the emission spot.

2. There is no significant difference between $2 \mathrm{D}$ and $3 \mathrm{D}$ models. Assuming the same total emission current, reflected power and transmitted rf power behave similarly in both models.

3. Electron current from the spot spreads over the inside surface of the cell, but the major part of the emitted current goes to a small area on an iris opposite to the emitting spot and to a current returning back to the emitting spot.

4. Secondary or back-scattered electrons do not change significantly the behavior of the rf fields.

\subsection{Standing wave}

Breakdown behavior of standing wave (SW) structures $[1,2]$ is very different from TW structures. In the TW case, a major part of the rf energy is absorbed by breakdown currents; in the SW case rf energy is reflected from the structure. After breakdown starts, reflected energy increases during $\sim 100 \mathrm{~ns}$ in TW case, and in $\sim 10 \mathrm{~ns}$ in SW case. We simulated a 2D SW structure to find the source of these differences. We used dimensions of the $\pi$ phase advance $S W$ structure that is currently under high power test at SLAC [5]. The simulated reflected power and a signal from a field probe are shown in Fig. 2. The main results of these simulations are listed below:

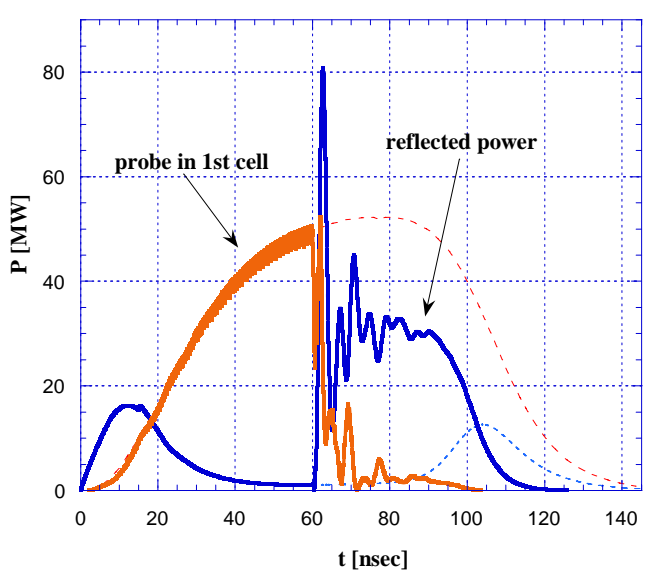

Figure 2: Reflected power and power from a simulated probe in the structure $v s$. time. Power from the probe is arbitrarily normalized. Emission starts at $60 \mathrm{~ns}$. Dashed curves - no emission, solid curves - with emission.

1. Electromagnetic fields in the structure collapse just after emission starts. Currents pass across the whole cavity and absorb a major part of the stored rf energy in a few nanoseconds, compared to a filling time of $\sim 100 \mathrm{~ns}$.

2. The currents detune the whole structure, causing the $\pi$-resonance to shift from the working frequency. This shift causes rf energy to reflect from input iris of the structure.

3. The increase of the electric field in the cells (due to emitted currents) is generally smaller than for the TW structure.

The main difference between the TW and SW case is in the coupling of the structure cavity to the input waveguide. The goal in TW coupler design is to have a small reflection from the coupler over a wide frequency range $\left(\frac{\Delta f}{f} \sim 10^{-3}\right)$. In the SW case, the goal is a small reflection from the beamloaded structure in a narrow frequency range $\left(\frac{\Delta f}{f} \sim 10^{-4}\right)$. In the TW case breakdown currents on the order of $10 \mathrm{~A}$ have a negligible effect on transmission and reflection of $\mathrm{rf}$ power, but in the SW case the same current shifts the resonant frequency enough to cause reflection of a major part of the incident rf power. We think that this high sensitivity of the SW structure to the breakdown currents may explain why the SW structures have reached higher maximum gradients than TW structures.

\section{REFERENCES}

[1] G.A. Loew and J.W. Wang, SLAC-PUB-4647 (1988).

[2] G.A. Loew and J.W. Wang, XIVth Int. Symp. on Disch. and Elec. Ins. in Vacuum, Santa Fe, New Mexico, September 16-20, 1990.

[3] J.W. Wang at al, "High Gradient Tests of SLAC Linear Collider Accelerating Structures," SLAC-PUB-6617 (1994).

[4] C. Adolphsen at al, "RF Processing of X-Band Accelerator Structures at the NLCTA," LINAC2000, August, 2000, Monterey, Ca.

[5] C. Adolphsen at al, Paper ROAA003 this conference.

[6] http://www.mrcwdc.com/Magic/ 